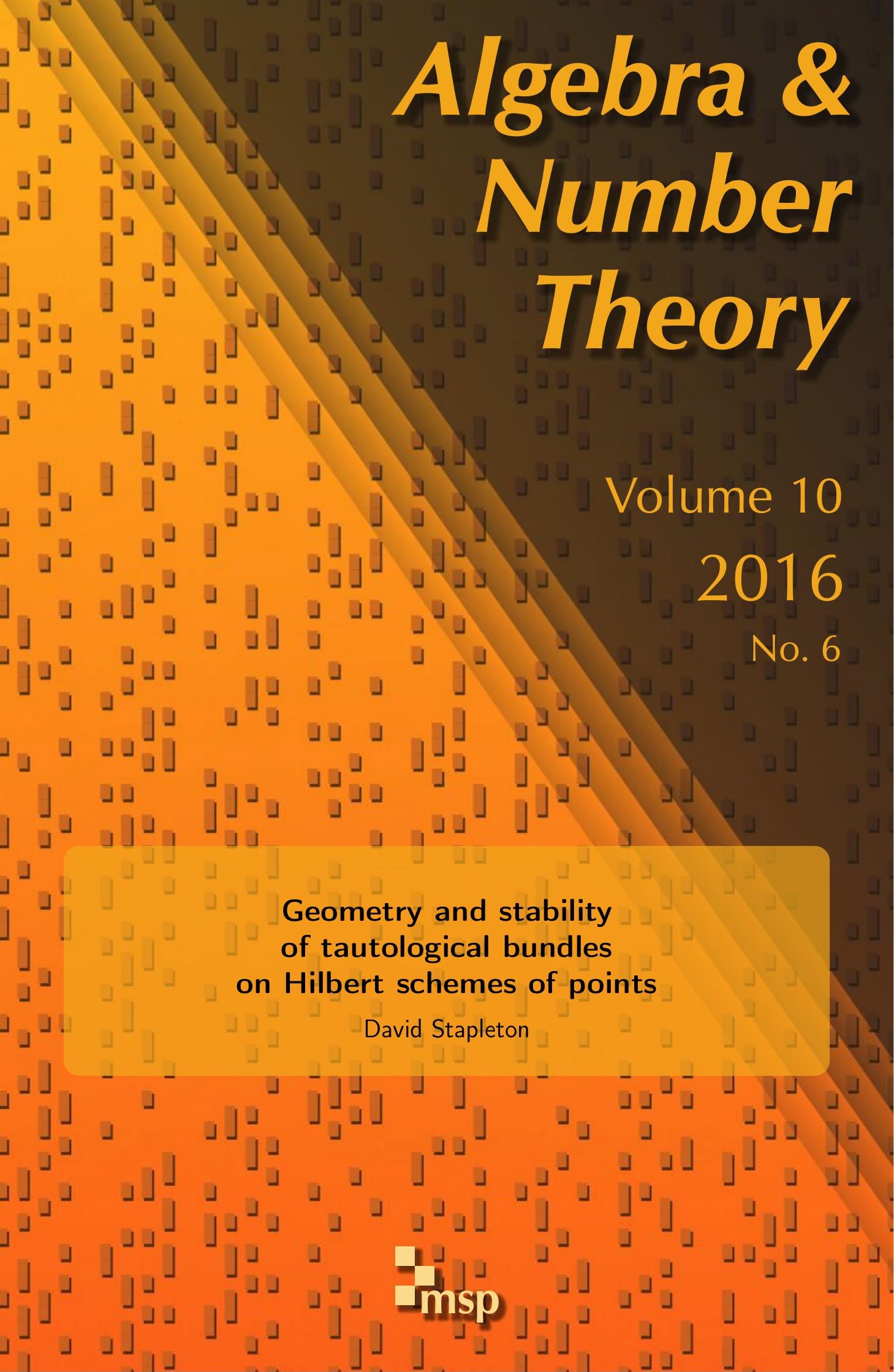




\title{
Geometry and stability of tautological bundles on Hilbert schemes of points
}

\author{
David Stapleton
}

\begin{abstract}
We explore the geometry and establish the slope-stability of tautological vector bundles on Hilbert schemes of points on smooth surfaces. By establishing stability in general, we complete a series of results of Schlickewei and Wandel, who proved the slope-stability of these vector bundles for Hilbert schemes of 2 points or 3 points on $\mathrm{K} 3$ or abelian surfaces with Picard group restrictions. In exploring the geometry, we show that every sufficiently positive semistable vector bundle on a smooth curve arises as the restriction of a tautological vector bundle on the Hilbert scheme of points on the projective plane. Moreover, we show that the tautological bundle of the tangent bundle is naturally isomorphic to the log tangent sheaf of the exceptional divisor of the Hilbert-Chow morphism.
\end{abstract}

\section{Introduction}

The purpose of this paper is to explore the geometry of tautological bundles on Hilbert schemes of smooth surfaces and to establish the slope-stability of these bundles.

Let $S$ be a smooth complex projective surface, and denote by $S^{[n]}$ the Hilbert scheme parametrizing length- $n$ subschemes of $S$. This parameter space carries some natural tautological vector bundles: if $\mathcal{L}$ is a line bundle on $S$ then $\mathcal{L}^{[n]}$ is the rank- $n$ vector bundle whose fiber at the point corresponding to a length- $n$ subscheme $\xi \subset S$ is the vector space $H^{0}\left(S, \mathcal{L} \otimes \mathcal{O}_{\xi}\right)$. These tautological vector bundles have attracted a great deal of interest. Lehn [1999] first computed the cohomology of the tautological bundles. Later Danila [2001] and Scala [2009] identified the induced symmetric group representations on the cohomology of the tautological bundles. Ellingsrud and Strømme [1993] showed that the Chern classes of the bundles $\mathcal{O}_{\mathbb{P} 2}^{[n]}, \mathcal{O}_{\mathbb{P}^{2}}(1)^{[n]}$, and $\mathcal{O}_{\mathbb{P}^{2}}(2)^{[n]}$ generate the cohomology of $\left(\mathbb{P}^{2}\right)^{[n]}$. Nakajima gave a nicely exposited interpretation $[1999, \S 4.3]$ of the McKay correspondence by

MSC2010: 14J60.

Keywords: Hilbert schemes of surfaces, vector bundles on surfaces, Fourier-Mukai transforms, slope-stability, spectral curves, log tangent bundle, tautological bundles, Hilbert schemes of points. 
restricting the tautological bundles to the $G$-Hilbert scheme. Recently Okounkov [2014] formulated a conjecture about special generating functions associated to the tautological bundles.

Given the importance of the tautological bundles, it is natural to explore how different geometric aspects of vector bundles transform to their tautological bundles. For instance, we ask when the tautological bundle of a stable bundle is also stable. In [Schlickewei 2010; Wandel 2013; 2014] this question has been answered positively for Hilbert schemes of 2 points or 3 points on a K3 or abelian surface with Picard group restrictions. Our first result establishes the stability of these bundles for arbitrary $n$ and any surface.

Theorem A. If $\mathcal{L}$ is a nontrivial line bundle on $S$, then $\mathcal{L}^{[n]}$ is slope-stable with respect to natural Chow divisors on $S^{[n]}$.

More precisely, an ample divisor on $S$ determines a natural ample divisor on $\operatorname{Sym}^{n}(S)$, and the pullback via the Hilbert-Chow morphism gives one such natural Chow divisor on $S^{[n]}$, which is not ample but is big and semiample. More generally, we prove that if $\mathcal{E} ¥ \mathcal{O}_{S}$ is any slope-stable vector bundle on $S$ with respect to some ample divisor then $\mathcal{E}^{[n]}$ is slope-stable with respect to the corresponding Chow divisor. Although Theorem A only gives stability with respect to a strictly big and nef divisor, we are able to deduce stability with respect to nearby ample divisors via a perturbation argument on the nef cone.

If $S$ is any smooth surface, there is a divisor $B_{n}$ in $S^{[n]}$ which consists of nonreduced subschemes. The pair $\left(S^{[n]}, B_{n}\right)$ gives a natural closure of the space of $n$ distinct points in $S$. The vector fields on $S^{[n]}$ tangent to $B_{n}$ form the sheaf of logarithmic vector fields $\operatorname{Der}_{\mathbb{C}}\left(-\log B_{n}\right)$. Our second result says the sheaf $\operatorname{Der}_{\mathbb{C}}\left(-\log B_{n}\right)$ is naturally isomorphic to the tautological bundle associated to the tangent bundle on $S$.

Theorem B. For any smooth surface $S$ there exists a natural injection

$$
\alpha_{n}:\left(T_{S}\right)^{[n]} \rightarrow T_{S^{[n]}},
$$

and $\alpha_{n}$ induces an isomorphism between $\left(T_{S}\right)^{[n]}$ and $\operatorname{Der}_{\mathbb{C}}\left(-\log B_{n}\right)$.

The analogous statement also holds for smooth curves. In general, the sheaves $\operatorname{Der}_{\mathbb{C}}\left(-\log B_{n}\right)$ are only guaranteed to be reflexive, as $B_{n}$ is not a simple normal crossing divisor. However, Theorem B shows $\operatorname{Der}_{\mathbb{C}}\left(-\log B_{n}\right)$ is locally free; that is, $B_{n}$ is a free divisor. Buchweitz, Ebeling, and Graf von Bothmer [Buchweitz et al. 2009] have already shown that $B_{n}$ is a free divisor using different methods.

Using Aubin and Yau's theorem [Aubin 1976] we obtain:

Corollary C. If a surface $S$ has ample canonical bundle, then the log tangent bundle $\operatorname{Der}_{\mathbb{C}}\left(-\log B_{n}\right)$ is polystable with respect to the big and nef canonical divisor $K_{S^{[n]}}$. 
Finally, we explore the geometry of the tautological bundles when the surface is the projective plane. We prove that the tautological bundles on $\left(\mathbb{P}^{2}\right)^{[n]}$ are rich enough to capture all semistable rank- $n$ bundles on curves.

Theorem D. If $C$ is a smooth projective curve and $\mathcal{E}$ is a semistable rank- $n$ vector bundle on $C$ with sufficiently positive degree, then there exists an embedding $C \rightarrow\left(\mathbb{P}^{2}\right)^{[n]}$ such that

$$
\left.\mathcal{O}_{\mathbb{P}^{2}}(1)^{[n]}\right|_{C} \cong \mathcal{E}
$$

The proof of Theorem A follows the approach taken by Mistretta [2006], who studies the stability of tautological bundles on the symmetric powers of a curve. The idea is to examine the tautological vector bundles on the cartesian power $S^{n}$ and show there are no $\mathfrak{S}_{n}$-equivariant destabilizing subsheaves. This strategy is more effective for surfaces because the diagonals in $S^{n}$ have codimension 2. The map in Theorem B arises from pushing forward the normal sequence of the universal family. The proof of Theorem D is constructive, using the spectral curves of Beauville, Narasimhan, and Ramanan [Beauville et al. 1989].

In Section 1 we give the proof of Theorem A. In Section 2 we prove Theorem B and deduce Corollary C. In Section 3 we prove Theorem D. In Section 4 we give the perturbation argument, deducing that the tautological bundles are stable with respect to ample divisors.

Throughout, we work over the complex numbers. If $X$ is a variety of dimension $d$ and $\mathcal{E}$ is a vector bundle on $X$, then for any divisor class $H \in N^{1}(X)$ we define the slope of $\mathcal{E}$ with respect to $H$ to be the rational number

$$
\mu_{H}(\mathcal{E}):=\frac{c_{1}(\mathcal{E}) \cdot H^{d-1}}{\operatorname{rank}(\mathcal{E})} .
$$

We say $\mathcal{E}$ is slope-stable (resp. slope-semistable) with respect to $H$ if, for all subsheaves $\mathcal{F} \subset \mathcal{E}$ of intermediate rank, we have

$$
\mu_{H}(\mathcal{F})<\mu_{H}(\mathcal{E}) \quad\left(\text { resp. } \mu_{H}(\mathcal{F}) \leq \mu_{H}(\mathcal{E})\right)
$$

\section{Stability of tautological bundles}

In this section we prove that the tautological bundle of a stable vector bundle $\mathcal{E}$ is stable with respect to natural Chow divisors on $S^{[n]}$. Thus we deduce Theorem A when $\mathcal{E}$ is a nontrivial line bundle. We start by defining the essential objects in the study of Hilbert schemes of points on surfaces.

Let $S$ be a smooth complex projective surface. We write $S^{[n]}$ for the Hilbert scheme of length- $n$ subschemes of $S$. We denote by $\mathcal{Z}_{n}$ the universal family of $S^{[n]}$ 
with the following projections:

$$
S \times S^{[n]} \underset{p_{2} \downarrow}{\mathcal{Z}_{n} \stackrel{p_{1}}{\longrightarrow} S} \underset{S^{[n]}}{\longrightarrow}
$$

For a fixed vector bundle $\mathcal{E}$ on $S$ of rank $r$, we define

$$
\mathcal{E}^{[n]}:=\left(p_{2}\right)_{*}\left(p_{1}^{*} \mathcal{E}\right),
$$

which is the tautological vector bundle associated to $\mathcal{E}$ and has rank $r n$. The fiber of $\mathcal{E}^{[n]}$ at a point $[\xi] \in S^{[n]}$ can be naturally identified with the vector space $H^{0}\left(S,\left.\mathcal{E}\right|_{\xi}\right)$.

The symmetric group on $n$ elements, $\mathfrak{S}_{n}$, naturally acts on the cartesian product $S^{n}$, and we write $\sigma_{n}$ for the quotient map

$$
\sigma_{n}: S^{n} \rightarrow S^{n} / \mathfrak{S}_{n}=: \operatorname{Sym}^{n}(S) .
$$

There is also a Hilbert-Chow morphism,

$$
h_{n}: S^{[n]} \rightarrow \operatorname{Sym}^{n}(S),
$$

which is a semismall map [de Cataldo and Migliorini 2002, Definition 2.1.1].

We wish to view $\mathcal{E}^{[n]}$ as an $\mathfrak{S}_{n}$-equivariant sheaf on $S^{n}$. Recall that if $G$ is a finite group that acts on a scheme $X$, and if $\mathcal{F}$ is a coherent sheaf on $X$, then a $G$-equivariant structure on $\mathcal{F}$ is given by a choice of isomorphisms

$$
\phi_{g}: \mathcal{F} \rightarrow g^{*} \mathcal{F}
$$

for all $g \in G$ satisfying the compatibility condition $h^{*}\left(\phi_{g}\right) \circ \phi_{h}=\phi_{g h}$. Following [Danila 2001] and [Scala 2009], we study the tautological bundles on $S^{[n]}$ by working with $\mathfrak{S}_{n}$-equivariant sheaves on $S^{n}$. For our purposes it is enough to study $\mathcal{E}^{[n]}$ equivariantly on the open subset of distinct points in $S^{[n]}$.

We write $\operatorname{Sym}^{n}(S)$ 。 for the open subset of $\operatorname{Sym}^{n}(S)$ of distinct points. Likewise, given a map $f: X \rightarrow \operatorname{Sym}^{n}(S)$, we write $X_{\circ}$ for $f^{-1}\left(\operatorname{Sym}^{n}(S)_{\circ}\right)$. By abuse of notation, given another map $g: X \rightarrow Y$ with domain $X$ we define $g_{\circ}:=\left.g\right|_{X_{\circ}}$, and given a coherent sheaf $\mathcal{F}$ on $X$ we define $\mathcal{F}_{\circ}:=\left.\mathcal{F}\right|_{X_{\circ}}$. The map $h_{n, \circ}: S_{\circ}^{[n]} \rightarrow \operatorname{Sym}^{n}(S)_{\circ}$ is an isomorphism. We define

$$
\bar{\sigma}_{n, \circ}:=h_{n, \circ}^{-1} \circ \sigma_{n, \circ}: S_{\circ}^{n} \rightarrow S_{\circ}^{[n]} .
$$

Given a torsion-free coherent sheaf $\mathcal{F}$ on $S^{[n]}$, we define a torsion-free coherent sheaf on $S^{n}$ by

$$
(\mathcal{F})_{S^{n}}:=j_{*}\left(\bar{\sigma}_{n, \circ}^{*}\left(\mathcal{F}_{\circ}\right)\right),
$$

where $j$ is the inclusion $j: S_{\circ}^{n} \rightarrow S^{n}$. The sheaf $(\mathcal{F})_{S^{n}}$ can be thought of as a modification of $\mathcal{F}$ along the exceptional divisor of $h_{n}$. 
The pullback $\bar{\sigma}_{n, \circ}^{*}(-)$ is left exact, as the map $\bar{\sigma}_{n, \circ}$ is étale; thus the functor $(-)_{S^{n}}$ is left exact. If $\mathcal{F}$ is reflexive, the normality of $S^{n}$ implies that the natural $\mathfrak{S}_{n}$-equivariant structure on the reflexive sheaf $\bar{\sigma}_{n, 0}^{*}\left(\mathcal{F}_{\circ}\right)$ pushes forward uniquely to an $\mathfrak{S}_{n}$-equivariant structure on $(\mathcal{F})_{S^{n}}$.

Let $q_{i}$ denote the projection from $S^{n}$ onto the $i$-th factor. Given a vector bundle $\mathcal{E}$ on $S$, there is an $\mathfrak{S}_{n}$-equivariant vector bundle on $S^{n}$ defined by

$$
\mathcal{E}^{\boxplus n}:=\bigoplus_{i=1}^{n} q_{i}^{*}(\mathcal{E}) .
$$

We have given two natural $\mathfrak{S}_{n}$-equivariant sheaves on $S^{n}$ associated to $\mathcal{E}$. In fact, they are equivalent.

Lemma 1.1. Given a vector bundle $\mathcal{E}$ on $S$ there is an isomorphism

$$
\left(\mathcal{E}^{[n]}\right)_{S^{n}} \cong \mathcal{E}^{\boxplus n}
$$

of $\mathfrak{S}_{n}$-equivariant vector bundles on $S^{n}$.

Proof. Consider the following fiber square:

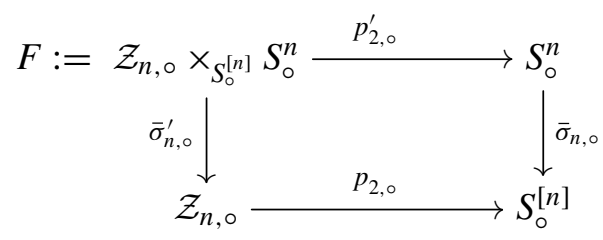

Every map in the fiber square is an étale map between $\mathfrak{S}_{n}$-schemes (the $\mathfrak{S}_{n}$-action on $\mathcal{Z}_{n, \circ}$ and $S_{\circ}^{[n]}$ is trivial). We write $\Gamma_{i}$ for the subscheme of $S_{\circ}^{n} \times S$ that is the graph of the map $q_{i, \circ}: S_{\circ}^{n} \rightarrow S$. The scheme $F$ is equal to the disjoint union $\bigsqcup \Gamma_{i}$ and is a subscheme of $S_{\circ}^{n} \times S$. The restriction $\left.p_{1, \circ} \circ \bar{\sigma}_{n, o}^{\prime}\right|_{\Gamma_{i}}$ is the projection $\Gamma_{i} \rightarrow S$. So there is an equivariant isomorphism

$$
\left(p_{2, \mathrm{o}}^{\prime}\right)_{*}\left(\left(\bar{\sigma}_{n, \circ}^{\prime}\right)^{*}\left(p_{1, \mathrm{o}}^{*}(\mathcal{E})\right)\right) \cong \mathcal{E}_{\circ}^{\boxplus n} .
$$

As the fiber square is made of flat proper $\mathfrak{S}_{n}$-maps, there is a natural $\mathfrak{S}_{n}$ equivariant isomorphism

$$
\left(p_{2, \mathrm{o}}^{\prime}\right)_{*}\left(\left(\bar{\sigma}_{n, \mathrm{o}}^{\prime}\right)^{*}\left(p_{1, \mathrm{o}}^{*}(\mathcal{E})\right)\right) \cong \bar{\sigma}_{n, \mathrm{o}}^{*}\left(\left(p_{2, \mathrm{o}}\right)_{*}\left(p_{1, \mathrm{o}}^{*}(\mathcal{E})\right)\right) .
$$

The latter sheaf is $\left(\mathcal{E}^{[n]}\right)_{S^{n}, \circ}$. Finally, any isomorphism between vector bundles on $S_{\circ}^{n}$ uniquely extends to an isomorphism between their pushforwards along $j$. Therefore, there is a natural $\mathfrak{S}_{n}$-equivariant isomorphism $\left(\mathcal{E}^{[n]}\right)_{S^{n}} \cong \mathcal{E}^{\boxplus n}$. 
Given an ample divisor $H$ on $S$, there is a natural $\mathfrak{S}_{n}$-invariant ample divisor on $S^{n}$ defined by

$$
H_{S^{n}}:=\sum_{i=1}^{n} q_{i}^{*}(H)
$$

This is the Chow divisor that appears in Theorem A. Fogarty [1973, Lemma 6.1] shows every divisor $H_{S^{n}}$ descends to an ample Cartier divisor on $\operatorname{Sym}^{n}(S)$. Pulling back this Cartier divisor along the Hilbert-Chow morphism gives a big and nef divisor on $S^{[n]}$, which we denote by $H_{n}$. If $H$ is effective then $H_{n}$ can be realized set-theoretically as

$$
H_{n}=\left\{\xi \in S^{[n]} \mid \xi \cap \operatorname{Supp}(H) \neq \varnothing\right\} .
$$

Lemma 1.2. If $\mathcal{F}$ is a torsion-free sheaf on $S^{[n]}$ then

$$
(n !) \int_{S^{[n]}} c_{1}(\mathcal{F}) \cdot\left(H_{n}\right)^{2 n-1}=\int_{S^{n}} c_{1}\left((\mathcal{F})_{S^{n}}\right) \cdot\left(H_{S^{n}}\right)^{2 n-1} .
$$

Proof. This is a straightforward calculation using $S_{\circ}^{[n]}, \operatorname{Sym}^{n}(S)_{\circ}$, and $S_{\circ}^{n}$.

In the following lemma we assume Proposition 4.7, which says the pullback of a stable bundle to a product is stable with respect to a product polarization. For the sake of the exposition we give the proof of Proposition 4.7 in Section 4.

Lemma 1.3. If $\mathcal{E} ¥ \mathcal{O}_{S}$ is slope-stable on $S$ with respect to an ample divisor $H$ then there are no $\mathfrak{S}_{n}$-equivariant subsheaves of $\mathcal{E}^{\boxplus n}$ that are slope-destabilizing with respect to $H_{S^{n}}$.

Proof. Let $0 \neq \mathcal{F} \subset \mathcal{E}^{\boxplus n}$ be an $\mathfrak{S}_{n}$-equivariant subsheaf. We can find a (not necessarily equivariant) slope-stable subsheaf $0 \neq \mathcal{F}^{\prime} \subset \mathcal{F}$ which has maximal slope with respect to $H_{S^{n}}$. Fix $i$ so that the composition

$$
\mathcal{F}^{\prime} \rightarrow \mathcal{E}^{\boxplus n} \rightarrow q_{i}^{*} \mathcal{E}
$$

is nonzero. By Proposition 4.7 we know that each $q_{i}^{*} \mathcal{E}$ is slope-stable with respect to $H_{S^{n}}$. A nonzero map between slope-stable sheaves can only exist if

(1) the slope of $\mathcal{F}^{\prime}$ is less than the slope of $q_{i}^{*} \mathcal{E}$, or

(2) $\mathcal{F}^{\prime} \rightarrow q_{i}^{*} \mathcal{E}$ is an isomorphism.

In case $(1), \mu_{H_{S^{n}}}(\mathcal{F}) \leq \mu_{H_{S^{n}}}\left(\mathcal{F}^{\prime}\right)<\mu_{H_{S^{n}}}\left(q_{i}^{*} \mathcal{E}\right)$. By symmetry, $\mu_{H_{S^{n}}}\left(q_{i}^{*} \mathcal{E}\right)=$ $\mu_{H_{S^{n}}}\left(q_{j}^{*} \mathcal{E}\right)$ for all $i$ and $j$. Thus we have $\mu_{H_{S^{n}}}\left(q_{i}^{*} \mathcal{E}\right)=\mu_{H_{S^{n}}}\left(\mathcal{E}^{\boxplus n}\right)$, and $\mathcal{F}$ does not destabilize $\mathcal{E}^{\boxplus n}$. 
In case (2), we know $\mathcal{F}^{\prime} \cong q_{i}^{*} \mathcal{E}$. Because $\mathcal{E} \not \mathcal{O}_{S}$, the pullbacks $q_{i}^{*} \mathcal{E}$ and $q_{j}^{*} \mathcal{E}$ are not isomorphic unless $i=j$. As all the $q_{j}^{*} \mathcal{E}$ have the same slope and are stable with respect to $H_{S^{n}}$, we have $\operatorname{Hom}\left(\mathcal{F}^{\prime}, q_{j}^{*} \mathcal{E}\right)=0$ for $j \neq i$. In particular, all the compositions

$$
\mathcal{F}^{\prime} \rightarrow \mathcal{E}^{\boxplus n} \rightarrow q_{j}^{*} \mathcal{E}
$$

are zero for $j \neq i$. Thus $\mathcal{F}^{\prime}$ is a summand of $\mathcal{E}^{\boxplus n}$. So $\mathcal{F}$ is an $\mathfrak{S}_{n}$-equivariant subsheaf of $\mathcal{E}^{\boxplus n}$, which contains one of the summands. But $\mathfrak{S}_{n}$ acts transitively on the summands so $\mathcal{F}$ contains all the summands, hence $\mathcal{F}$ does not destabilize $\mathcal{E}^{\boxplus n}$.

Now we prove Theorem A in full generality.

Theorem 1.4. If $\mathcal{E} ¥ \mathcal{O}_{S}$ is a vector bundle on $S$ which is slope-stable with respect to an ample divisor $H$, then $\mathcal{E}^{[n]}$ is slope-stable with respect to $H_{n}$.

Proof. Let $\mathcal{F} \subset \mathcal{E}^{[n]}$ be a reflexive subsheaf of intermediate rank. It is enough to consider reflexive sheaves because the saturation of a torsion-free subsheaf of $\mathcal{E}^{[n]}$ is reflexive of the same rank and its slope cannot decrease. By Lemma 1.2, the slope of a torsion-free sheaf $\mathcal{F}$ with respect to $H_{n}$ is, up to a fixed positive multiple, the same as the slope of $(\mathcal{F})_{S^{n}}$ with respect to $H_{S^{n}}$. In particular,

$$
\mu_{H_{n}}(\mathcal{F})<\mu_{H_{n}}\left(\mathcal{E}^{[n]}\right) \Longleftrightarrow \mu_{H_{S^{n}}}\left((\mathcal{F})_{S^{n}}\right)<\mu_{H_{S^{n}}}\left(\mathcal{E}^{\boxplus n}\right) .
$$

Now $(\mathcal{F})_{S^{n}}$ is naturally an $\mathfrak{S}_{n}$-equivariant subsheaf of $\mathcal{E}^{\boxplus n}$. Thus, by Lemma 1.3 ,

$$
\mu_{H_{S^{n}}}\left((\mathcal{F})_{S^{n}}\right)<\mu_{H_{S^{n}}}\left(\mathcal{E}^{\boxplus n}\right) .
$$

Therefore, $\mu_{H_{n}}(\mathcal{F})<\mu_{H_{n}}\left(\mathcal{E}^{[n]}\right)$ for all torsion-free subsheaves of intermediate rank, and $\mathcal{E}^{[n]}$ is stable with respect to $H_{n}$.

\section{The tautological tangent map}

For any smooth (not necessarily projective) surface $S$, the Hilbert scheme $S^{[n]}$ is a smooth closure of the space of $n$ distinct points in $S$. The boundary $B_{n}$ is the locus of nonreduced length- $n$ subschemes of $S$. We are interested in vector fields which are tangent to the boundary $B_{n}$.

Definition 2.1. If $D$ is a codimension-1 subvariety of a smooth variety $X$, then the sheaf of logarithmic vector fields, denoted $\operatorname{Der}_{\mathbb{C}}(-\log D)$, is the subsheaf of $T_{X}$ consisting of vector fields which along the regular locus of $D$ are tangent to $D$.

When $D$ is smooth, $\operatorname{Der}_{\mathbb{C}}(-\log D)$ is just the elementary transformation of the tangent bundle along the normal bundle of $D$ in $X$; in particular, it is a vector bundle. Even when $D$ is singular, $\operatorname{Der}_{\mathbb{C}}(-\log D)$ is reflexive by definition, so it is enough to define $\operatorname{Der}_{\mathbb{C}}(-\log D)$ away from the singular locus (or any codimension-2 set in $X$ ) of $D$ and then pushforward. 
For Hilbert schemes of points on a surface, $\operatorname{Der}_{\mathbb{C}}\left(-\log B_{n}\right)$ can be naturally understood as the tautological bundle of the tangent bundle on the surface.

Theorem B. For any smooth connected surface $S$ there exists a natural injection

$$
\alpha_{n}:\left(T_{S}\right)^{[n]} \rightarrow T_{S^{[n]}},
$$

and $\alpha_{n}$ induces an isomorphism between $\left(T_{S}\right)^{[n]}$ and $\operatorname{Der}_{\mathbb{C}}\left(-\log B_{n}\right)$.

At a point $[\xi] \in S^{[n]}$ the map $\left.\alpha_{n}\right|_{[\xi]}$ can be interpreted as deformations of $\xi$ coming from tangent vectors of $S$. We expect that the degeneracy loci of $\alpha_{n}$ give an interesting stratification of $S^{[n]}$.

Before proving Theorem B we prove a general lemma.

Lemma 2.2. Let $X$ and $Y$ be smooth varieties and $f: X \rightarrow Y$ a branched covering with reduced branch locus $B \subset Y$. If $\delta \in H^{0}(Y, T Y)$ is a vector field on $Y$ whose pullback $f^{*} \delta \in H^{0}\left(X, f^{*} T Y\right)$ is in the image of

$$
d f: H^{0}(X, T X) \rightarrow H^{0}\left(X, f^{*} T Y\right),
$$

then $\delta \in H^{0}\left(Y, \operatorname{Der}_{\mathbb{C}}(-\log B)\right)$.

Proof. It is enough to check that $\delta$ is tangent to $B$ for points $p \in B$ outside of a codimension-2 subset in $Y$. Let $p \in B$ be a general point and $q$ a ramified point in the fiber of $f$ over $p$. We can choose local analytic coordinates $y_{1}, \ldots, y_{n}$ centered at $p$ and coordinates $x_{1}, \ldots, x_{n}$ centered at $q$ such that

$$
f^{*}\left(y_{1}\right)=x_{1}^{m}, \quad f^{*}\left(y_{i}\right)=x_{i} \quad \text { for } i>1 .
$$

That is, $y_{1}$ is a local equation for $B$ and $x_{1}$ is a local equation for the reduced component of ramification containing $q$. Then the derivative $d f$ maps

$$
\frac{\partial}{\partial x_{1}} \mapsto m x_{1}^{m-1} f^{*}\left(\frac{\partial}{\partial y_{1}}\right), \quad \frac{\partial}{\partial x_{i}} \mapsto f^{*}\left(\frac{\partial}{\partial y_{i}}\right) \text { for } i>1 \text {. }
$$

Now $f^{*} \delta$ is in the image of $d f$. Expanding locally,

$$
f^{*} \delta=f^{*}\left(g_{1}\right) f^{*}\left(\frac{\partial}{\partial y_{1}}\right)+\cdots+f^{*}\left(g_{n}\right) f^{*}\left(\frac{\partial}{\partial y_{n}}\right) .
$$

Thus $x_{1}^{m-1}$ divides $f^{*}\left(g_{1}\right)$. So $y_{1}$ divides $g_{1}$ and $\delta$ is in $H^{0}\left(Y, \operatorname{Der}_{\mathbb{C}}(-\log B)\right)$.

Proof of Theorem B. As in Section 1 we use $\mathcal{Z}_{n} \subset S \times S^{[n]}$ to denote the universal family of the Hilbert scheme of points. Applying relative Serre duality to the main result of [Lehn 1998] shows that the tangent bundle of $S^{[n]}$ is given by $T_{S^{[n]}}=\left(p_{2}\right)_{*} \mathcal{H} \operatorname{om}\left(\mathcal{I}_{\mathcal{Z}_{n}}, \mathcal{O}_{\mathcal{Z}_{n}}\right)$. The normal sequence for $\mathcal{Z}_{n}$ gives a map

$$
\left.p_{1}^{*} T_{S} \oplus p_{2}^{*} T_{S^{[n]}} \cong T_{S \times S^{[n]}}\right|_{\mathcal{Z}_{n}} \stackrel{\beta}{\rightarrow}\left(\mathcal{I}_{\mathcal{Z}_{n}} / \mathcal{I}_{\mathcal{Z}_{n}}^{2}\right)^{\vee} \cong \mathcal{H o m}\left(\mathcal{I}_{\mathcal{Z}_{n}}, \mathcal{O}_{\mathcal{Z}_{n}}\right) .
$$


Thus after pushing forward the first summand we get a map

$$
\alpha_{n}:\left(T_{S}\right)^{[n]}:=\left(p_{2}\right)_{*}\left(p_{1}^{*} T_{S}\right) \rightarrow\left(p_{2}\right)_{*} \mathcal{H o m}\left(\mathcal{I}_{\mathcal{Z}_{n}}, \mathcal{O}_{\mathcal{Z}_{n}}\right)=T_{S^{[n]}} .
$$

To prove that $\alpha_{n}$ maps $\left(T_{S}\right)^{[n]}$ isomorphically onto $\operatorname{Der}_{\mathbb{C}}\left(-\log B_{n}\right)$ we first restrict to the open set $U \subset S^{[n]}$ parametrizing subschemes $\xi \subset S$, where $\xi$ contains at least $n-1$ distinct points. The complement of $U$ has codimension 2 so by reflexivity it is enough to prove the theorem on $U$. Moreover, the open set

$$
V:=p_{2}^{-1} U \subset \mathcal{Z}_{n}
$$

is smooth so we are in a situation where we can apply Lemma 2.2. There is a map

$$
\begin{aligned}
& \left.p_{2}^{*}\left(T_{S}\right)^{[n]}\right|_{V} \\
& \left.\downarrow p_{2}^{*} \alpha_{n}\right|_{V} \oplus-\left.\phi\right|_{V} \\
& \left.\left.\left.\left.0 \longrightarrow T_{\mathcal{Z}_{n}}\right|_{V} \longrightarrow p_{2}^{*} T_{S^{[n]}}\right|_{V} \oplus p_{1}^{*} T_{S}\right|_{V} \stackrel{\beta}{\longrightarrow} \mathcal{H o m}\left(\mathcal{I}_{\mathcal{Z}_{n}}, \mathcal{O}_{\mathcal{Z}_{n}}\right)\right|_{V}
\end{aligned}
$$

in which $\phi$ is the natural map coming from pulling back a pushforward. The composition

$$
\beta \circ\left(\left.p_{2}^{*} \alpha_{n}\right|_{V} \oplus-\left.\phi\right|_{V}\right)
$$

is identically zero. Therefore, the pullback of each local section of $\left.\left(T_{S}\right)^{[n]}\right|_{U}$ lies in $\left.T_{\mathcal{Z}_{n}}\right|_{V}$. It follows from Lemma 2.2 that $\left(T_{S}\right)^{[n]}$ is contained in $\operatorname{Der}_{\mathbb{C}}\left(-\log B_{n}\right)$. Now we can think of $\alpha_{n}$ as having codomain $\operatorname{Der}_{\mathbb{C}}\left(-\log B_{n}\right)$. The map is an isomorphism of $\left(T_{S}\right)^{[n]}$ and $\operatorname{Der}_{\mathbb{C}}\left(-\log B_{n}\right)$ away from $B_{n}$ and they both have the same first Chern class. Therefore, $\alpha_{n}$ could only fail to be an isomorphism in codimension greater than 2. But both sheaves are reflexive, and any isomorphism between reflexive sheaves away from codimension 2 on a normal variety extends uniquely to an isomorphism on the whole variety.

Proof of Corollary C. As a reminder, a vector bundle is polystable if it is a direct sum of stable bundles of the same slope. The theorem of Aubin and Yau [Aubin 1976] proves the existence of Kähler-Einstein metrics for canonically polarized manifolds. This implies that the tangent bundle is polystable with respect to the canonical bundle (see [Kobayashi 1987, Theorem 8.3]; this is the easy direction of the Donaldson-Uhlenbeck-Yau theorem [Donaldson 1985]). Thus $T_{S}$ is either stable or a direct sum of line bundles of the same canonical degree. In the first case, Corollary $\mathrm{C}$ follows directly from Theorems $\mathrm{A}$ and $\mathrm{B}$.

For the second case, let $T_{S} \cong \mathcal{L}_{1} \oplus \mathcal{L}_{2}$. First we point out that taking tautological bundles respects direct sums; that is,

$$
(\mathcal{E} \oplus \mathcal{F})^{[n]} \cong \mathcal{E}^{[n]} \oplus \mathcal{F}^{[n]} .
$$


We then note that neither $\mathcal{L}_{1}$ nor $\mathcal{L}_{2}$ is trivial so their tautological bundles are stable by Theorem A. And if two line bundles on $S$ have equal degrees with respect to the canonical bundle then their tautological bundles also have equal degrees with respect to $K_{S^{[n]}}$. Thus, by Theorem B, $\operatorname{Der}_{\mathbb{C}}\left(-\log B_{n}\right)$ is a direct sum of stable bundles of the same slope with respect to $K_{S^{[n]}}$, proving Corollary C.

Remark 2.3 (on the rank of $\alpha_{n}$ ). The restriction of $\alpha_{n}$ to any point $[\xi] \in S^{[n]}$ is precisely the map from $H^{0}\left(S,\left.T_{S}\right|_{\xi}\right)$ to $\operatorname{Hom}\left(I_{\xi}, \mathcal{O}_{\xi}\right)$ in the normal sequence of $\xi \subset S$. In [Bejleri and Stapleton 2016] we relate the rank of $\alpha_{n}$ to the dimension of the tangent space of the fibers of the Hilbert-Chow morphism. In particular, we show that if $\xi \subset \mathbb{C}^{2}$ is cut out by monomials and $P_{\xi}$ denotes the fiber of the Hilbert-Chow morphism at $\xi$, then

$$
\operatorname{dim} T_{[\xi]} P_{\xi}=2 n-\operatorname{rank}\left(\left.\alpha_{n}\right|_{[\xi]}\right) .
$$

Moreover, we give an explicit combinatorial formula for computing $\operatorname{rank}\left(\left.\alpha_{n}\right|_{[\xi]}\right)$ at these monomial subschemes.

\section{Spectral curves and tautological bundles}

In this section we prove that every sufficiently positive, rank- $n$, semistable vector bundle on a smooth projective curve arises as the pullback of $\mathcal{O}_{\mathbb{P}^{2}}(1)^{[n]}$ along an embedding of the curve in $\left(\mathbb{P}^{2}\right)^{[n]}$. To prove the theorem we need the spectral curves of [Beauville et al. 1989]. For completeness, we recall the construction.

Let $\pi: D \rightarrow C$ be an $n: 1$ map between smooth irreducible projective curves and let $\mathcal{E}$ be an $\mathcal{O}_{C}$-module. If $D$ can be embedded into the total space

$$
\mathbb{L}:=\operatorname{Spec}_{\mathcal{O}_{C}}\left(\operatorname{Sym}^{\bullet}\left(\mathcal{L}^{\vee}\right)\right) \stackrel{\pi_{\Perp}}{\longrightarrow} C
$$

of a line bundle $\mathcal{L}$ on $C$, with $\pi=\left.\pi_{\mathbb{L}}\right|_{D}$, then this gives a presentation

$$
\pi_{*} \mathcal{O}_{D} \cong \operatorname{Sym}^{\bullet}\left(\mathcal{L}^{\vee}\right) /\left(x^{n}+s_{1} x^{n-1}+\cdots+s_{n}\right)
$$

for $x^{n}+s_{1} x^{n-1}+\cdots+s_{n} \in H^{0}\left(\mathbb{L},\left(\pi_{\mathbb{L}}^{*} \mathcal{L}\right)^{\otimes n}\right)$. Here we write $x \in H^{0}\left(\mathbb{L}, \pi_{\mathbb{L}}^{*}(\mathcal{L})\right)$ for the coordinate section of $\pi_{\mathbb{L}}^{*}(\mathcal{L})$. To give $\mathcal{E}$ the structure of a $\pi_{*} \mathcal{O}_{D^{-}}$-module we need to specify a multiplication map $m: \mathcal{E} \otimes \mathcal{L}^{-1} \rightarrow \mathcal{E}$ (equivalently $\mathcal{E} \rightarrow \mathcal{E} \otimes \mathcal{L}$ ) which satisfies the relation $m^{n}+s_{1} m^{n-1}+\cdots+s_{n}=0$.

Every $\mathcal{L}$-twisted endomorphism $m: \mathcal{E} \rightarrow \mathcal{E} \otimes \mathcal{L}$ has an associated $\mathcal{L}$-twisted characteristic polynomial which is a global section $p_{m}(x) \in H^{0}\left(\mathbb{L},\left(\pi_{\mathbb{L}}^{*} \mathcal{L}\right)^{\otimes n}\right)$. A global version of the Cayley-Hamilton theorem says that $m$ automatically satisfies its $\mathcal{L}$-twisted characteristic polynomial. In particular, if the zero set of $p_{m}(x)$ is $D$ then $\mathcal{E}$ can naturally be thought of as a $\pi_{*} \mathcal{O}_{D}$-module. Fixing $s \in H^{0}\left(\mathbb{L},\left(\pi_{\mathbb{L}}^{*} \mathcal{L}\right)^{\otimes n}\right)$, which cuts out the integral curve $D$, [Beauville et al. 1989, Proposition 3.6] gives 
the beautiful correspondence

$\left\{\mathcal{E} \stackrel{m}{\rightarrow} \mathcal{E} \otimes \mathcal{L} \mid \mathcal{E}\right.$ a vector bundle and $\left.p_{m}(x)=s\right\}$

$$
\stackrel{1: 1}{\longrightarrow}\{\text { invertible sheaves } \mathcal{M} \text { on } D\} \text {. }
$$

The correspondence going from right to left is given by taking the coordinate section of $\pi_{\mathbb{L}}^{*}(\mathcal{L})$, restricting to $D$, twisting by $\mathcal{M}$, and pushing forward along $\pi$.

To prove Theorem $\mathrm{D}$ we need the following key lemma, which provides sufficient conditions for when a section of $\mathcal{E} \operatorname{nd}(\mathcal{E}) \otimes \mathcal{L}$ produces a smooth spectral curve.

Key Lemma. If $C$ is a smooth connected genus-g curve, $\mathcal{E}$ is a rank-n semistable vector bundle on $C$, and $\mathcal{L}$ is an ample line bundle on $C$ with $\operatorname{deg}(\mathcal{L}) \geq 2 g$, then the spectral curve associated to a generic section of $\mathcal{E} \operatorname{nd}(\mathcal{E}) \otimes \mathcal{L}$ is smooth and irreducible.

The method of proof of the Key Lemma involves a standard analysis of the discriminant locus, where a section of $\mathcal{E} \mathrm{nd}(\mathcal{E}) \otimes \mathcal{L}$ has eigenvalues with multiplicity $\geq 2$. Before proving the Key Lemma, we show that Theorem D follows immediately.

Proof of Theorem $D$. Let $C$ be a smooth projective genus- $g$ curve and $\mathcal{E}$ a rank- $n$ semistable vector bundle on $C$. Let $\mathcal{L}$ be a line bundle on $C$ of degree $\geq 2 g$. By the Key Lemma, if

$$
m: \mathcal{E} \rightarrow \mathcal{E} \otimes \mathcal{L}
$$

is a general $\mathcal{L}$-twisted endomorphism then the resulting $\mathcal{L}$-twisted characteristic polynomial is smooth and irreducible.

Thus, by the correspondence $(\diamond)$ there is a line bundle $\mathcal{M}$ on $D$ such that $\pi_{*} \mathcal{M} \cong \mathcal{E}$. The genus of $D$ is $g_{D}=\left(\begin{array}{l}r \\ 2\end{array}\right) \operatorname{deg}(\mathcal{L})+n(g-1)+1$ and is independent of $\mathcal{E}$. However, the degree of $\mathcal{M}$ is $\operatorname{deg}(\mathcal{E})+\left(\begin{array}{l}r \\ 2\end{array}\right) \operatorname{deg}(\mathcal{L})$ and does depend on the degree of $\mathcal{E}$. In particular, if

$$
\operatorname{deg}(\mathcal{E}) \geq\left(\begin{array}{l}
r \\
2
\end{array}\right) \operatorname{deg}(\mathcal{L})+r(2 g-2)+3
$$

then $\mathcal{M}$ is very ample and three general sections of $\mathcal{M}$ give a map $\phi: D \rightarrow \mathbb{P}^{2}$ such that the induced maps $\pi \times \phi: D \rightarrow C \times \mathbb{P}^{2}$ and $\psi_{\pi, \phi}: C \rightarrow\left(\mathbb{P}^{2}\right)^{[n]}$ are embeddings. Under the embedding $\psi_{\pi, \phi}$, the restriction of $\mathcal{O}_{\mathbb{P}^{2}}(1)^{[n]}$ to $C$ is precisely $\mathcal{E}$, proving Theorem D.

We now proceed with the proof of the Key Lemma.

Lemma 3.1. If a subvariety $X \subset \mathbb{E}$ of a globally generated vector bundle $\mathbb{E}$ over a smooth curve $C$ has codimension $\geq 2$ then a generic section of $\mathbb{E}$ avoids $X$. If $X \subset \mathbb{E}$ is a reduced divisor then a generic section of $\mathbb{E}$ meets $X$ transversely. 
Proof. This is an elementary dimension count using generic smoothness in characteristic 0 and the incidence correspondence

$$
I=\left\{\left(w, e_{x}, x\right) \in W \times\left.\mathbb{E}\right|_{x} \times C \mid w(x)=e_{x}\right\} \subset W \times \mathbb{E},
$$

where $W$ is a subspace of sections of $\mathbb{E} \rightarrow C$ that globally generate $\mathbb{E}$. The key point is that the projection from $I$ to $\mathbb{E}$ is an affine bundle, so the total space of $I$ is smooth.

If $\mathbb{W}$ is the total space of $\mathcal{E} \operatorname{nd}(\mathcal{E}) \otimes \mathcal{L}$, and $\mathcal{C}=\mathbb{L} \oplus \cdots \oplus \mathbb{L}^{\otimes n}$, then there is a map $\epsilon: \mathbb{H} \rightarrow \mathcal{C}$ which sends an $\mathcal{L}$-twisted endomorphism to the coefficients of its characteristic polynomial. There is a reduced and irreducible divisor in $\mathbb{U} \subset \mathcal{C}$ which consists of characteristic polynomials with multiple roots. Let $\mathbb{Q} \subset \mathbb{W}$ be the scheme-theoretic inverse of $\mathbb{U}$.

Lemma 3.2. $\mathbb{V}$ is reduced and irreducible. If a section $s: C \rightarrow \mathbb{W}$ meets $\mathbb{V}$ transversely and avoids the locus in $\mathbb{V}$ with more than one repeated eigenvalue or an eigenvalue of multiplicity $\geq 3$, then the corresponding spectral curve is smooth.

Proof. First, local trivialization of $\mathbb{U}, \mathbb{U}, \mathbb{V}$ and $\mathbb{Q}$ implies it is enough to check on a fiber. Over a point $x \in C$ we have $\left.\mathbb{H}\right|_{x} \cong \operatorname{Mat}_{n \times n}(k)$ and $\left.\mathcal{C}\right|_{x} \cong \mathbb{A}^{n}$. Let $\left.\mathbb{V}\right|_{x}$ be the locus of matrices whose eigenvalues have multiplicity $\geq 2$, and let $\left.\mathbb{U}\right|_{x}$ be the discriminant locus. Irreducibility of $\left.\mathbb{V}\right|_{x}$ follows from [Arnold 1971, §5.6], and the fact that it is reduced follows from the observation that $\left.d \epsilon\right|_{x, M}$ has maximal rank for a general matrix $\left.M \in \mathbb{U}\right|_{x}$. For the last statement in the lemma, it suffices to verify smoothness for an eigenvalues cover associated to a 1-dimensional family of matrices which meets the discriminant locus transversely at matrices with exactly one repeated eigenvalue; this is a straightforward local calculation.

Proof of Key Lemma. Semistability of $\mathcal{E}$ and the inequality $\operatorname{deg} \mathcal{L} \geq 2 g$ imply that $\mathcal{E}$ nd $(\mathcal{E}) \otimes \mathcal{L}$ is globally generated. By Lemma 3.1 and the first part of Lemma 3.2, a generic section $s$ of $\mathcal{E}$ nd $(\mathcal{E}) \otimes \mathcal{L}$ meets $\mathbb{V}$ transversely and avoids the locus with more than one repeated eigenvalue or an eigenvalue of multiplicity of $\geq 3$. By the second part of Lemma 3.2, the associated spectral curve is smooth. By construction of the spectral curve $C_{s}$ we have

$$
\pi_{*} \mathcal{O}_{C_{s}} \cong \mathcal{O}_{C} \oplus \cdots \oplus \mathcal{L}^{-(n-1)} .
$$

Since we assumed $\mathcal{L}$ is ample, $H^{0}\left(C_{s}, \mathcal{O}_{C_{s}}\right)=H^{0}\left(C, \pi_{*} \mathcal{O}_{C_{s}}\right)=H^{0}\left(C, \mathcal{O}_{C}\right)$ is 1-dimensional. Thus $C_{s}$ is connected and smooth, so it is irreducible.

\section{Perturbation of polarization and stability}

The goal of this section is to prove (in Proposition 4.7) that the pullback of a stable bundle to a product is stable with respect to a product polarization. Proposition 4.7 
was important in the proof of Theorem A. We also prove that stability of the tautological bundles with respect to the natural Chow divisors implies stability with respect to nearby ample divisors. Our approach to proving both of these facts involves considering stability with respect to numerical classes of curves so that we can apply ideas of convexity. In particular, our approach follows ideas appearing recently in [Greb and Toma 2013; Greb et al. 2016] and we recommend looking at these articles to see how these ideas can be developed further and systematically.

Throughout this section, denote by $\mathrm{X}$ a normal complex projective variety of dimension $d$. Let $\gamma \in N_{1}(X)_{\mathbb{R}}$ be a real curve class and let $\mathcal{E}$ be a torsion-free sheaf on X. For any sheaf $\mathcal{Q}$ on $X$, we denote by $\operatorname{Sing}(\mathcal{Q})$ the closed locus where $\mathcal{Q}$ is not locally free.

Definition 4.1. The slope of $\mathcal{E}$ with respect to $\gamma$ is the real number

$$
\mu^{\gamma}(\mathcal{E}):=\frac{c_{1}(\mathcal{E}) \cdot \gamma}{\operatorname{rank}(\mathcal{E})}
$$

Remark 4.2. Fixing an ample class $H \in N^{1}(X)_{\mathbb{R}}$, it is true that $\mu_{H}(\mathcal{E})=\mu^{H^{d-1}}(\mathcal{E})$. Nonetheless, to distinguish the concepts we use subscripts to denote slope with respect to an ample divisor and superscripts to denote slope with respect to a curve class.

Definition 4.3. We say $\mathcal{E}$ is slope-stable (resp. slope-semistable) with respect to $\gamma$ if, for all torsion-free quotients $\mathcal{E} \rightarrow \mathcal{Q} \rightarrow 0$ of intermediate rank, we have

$$
\mu^{\gamma}(\mathcal{E})<\mu^{\gamma}(\mathcal{Q}) \quad\left(\text { resp. } \mu^{\gamma}(\mathcal{E}) \leq \mu^{\gamma}(\mathcal{Q})\right) .
$$

A benefit of working with slope-(semi)stability with respect to curves rather than divisors is that we can apply ideas of convexity.

Lemma 4.4. If $\gamma, \delta$ are classes in $N_{1}(X)_{\mathbb{R}}$ such that $\mathcal{E}$ is semistable with respect to $\gamma$ and $\mathcal{E}$ is stable with respect to $\delta$, then $\mathcal{E}$ is stable with respect to $a \gamma+b \delta$ for $a, b>0$.

If $C \subset X$ is an irreducible curve, we would like to relate the stability of $\left.\mathcal{E}\right|_{C}$ and the stability of $\mathcal{E}$ with respect to the class of $C$. However, if $\mathcal{Q}$ is a coherent sheaf and $C$ meets $\operatorname{Sing}(\mathcal{Q})$, it is possible that $c_{1}\left(\left.\mathcal{Q}\right|_{C}\right) \neq\left. c_{1}(\mathcal{Q})\right|_{C}$. Thankfully we can say something if $C$ is not entirely contained in $\operatorname{Sing}(\mathcal{Q})$.

Proposition 4.5. Let $\mathcal{E} \rightarrow \mathcal{Q} \rightarrow 0$ be a torsion-free quotient which destabilizes $\mathcal{E}$ with respect to the curve class $\gamma$. Suppose $C \subset X$ is a smooth irreducible closed curve which represents $\gamma$, avoids $\operatorname{Sing}(\mathcal{E})$, and avoids the singularities of $X$. If $C$ is not contained in $\operatorname{Sing}(Q)$ then $\left.\mathcal{E}\right|_{C}$ is not stable on $C$. 
Proof. First, we can reduce to the surface case by choosing a normal surface $S \subset X$ containing $C$ such that $S$ is smooth along $C$, and $S$ meets $\operatorname{Sing}(\mathcal{Q})$ and $\operatorname{Sing}(\mathcal{E})$ properly. This is possible because when the dimension of $X$ is greater than 3 a generic, high-degree hyperplane section containing $C$ is normal and smooth along $C$ and meets both $\operatorname{Sing}(\mathcal{Q})$ and $\operatorname{Sing}(\mathcal{E})$ properly. Once such a surface is chosen, we have $\left.c_{1}(\mathcal{Q})\right|_{S}=c_{1}\left(\left.\mathcal{Q}\right|_{S}\right)=c_{1}\left(\left.\mathcal{Q}\right|_{S} / \operatorname{Tors}\left(\left.\mathcal{Q}\right|_{S}\right)\right),\left.\quad c_{1}(\mathcal{E})\right|_{S}=c_{1}\left(\left.\mathcal{E}\right|_{S}\right)=c_{1}\left(\left.\mathcal{E}\right|_{S} / \operatorname{Tors}\left(\left.\mathcal{E}\right|_{S}\right)\right)$ because both $\operatorname{Sing}(\mathcal{Q}) \cap S$ and $\operatorname{Sing}(\mathcal{E}) \cap S$ are zero-dimensional. Thus

$$
\left.\mathcal{E}\right|_{S} /\left.\operatorname{Tors}\left(\left.\mathcal{E}\right|_{S}\right) \rightarrow \mathcal{Q}\right|_{S} / \operatorname{Tors}\left(\left.\mathcal{Q}\right|_{S}\right) \rightarrow 0
$$

is a torsion-free quotient on $\mathrm{S}$ which destabilizes $\left.\mathcal{E}\right|_{S} / \operatorname{Tors}\left(\left.\mathcal{E}\right|_{S}\right)$ with respect to the class of $C$. So we have reduced the proposition to the case when $X$ is a surface.

Let $X$ be a surface. It is enough to show $c_{1}\left(\left.\mathcal{Q}\right|_{C}\right)=\left.c_{1}(\mathcal{Q})\right|_{C}$. The restriction $\left.c_{1}(\mathcal{Q})\right|_{C}$ is computed via the derived pullback

$$
\left.c_{1}(\mathcal{Q})\right|_{C}=\sum_{i=0}^{\infty}(-1)^{i} c_{1}\left(\operatorname{Tor}_{i}^{\mathcal{O}_{X}}\left(\mathcal{Q}, \mathcal{O}_{C}\right)\right),
$$

where the $\operatorname{Tor}_{i}^{\mathcal{O}_{X}}\left(\mathcal{Q}, \mathcal{O}_{C}\right)$ are thought of as modules on $C$ (see [Fulton 1998, §15.1] for the smooth case). Further, $C$ is a Cartier divisor on $X$, so $\mathcal{O}_{C}$ has a two-term locally free resolution. So the $\operatorname{Tor}_{i}^{\mathcal{O}_{X}}\left(\mathcal{Q}, \mathcal{O}_{C}\right)$ vanish for $i>2$ and $\operatorname{Tor}_{1}^{\mathcal{O}_{X}}\left(\mathcal{Q}, \mathcal{O}_{C}\right)=0$ because $\mathcal{Q}$ is torsion-free. Therefore,

$$
\left.c_{1}(\mathcal{Q})\right|_{C}=c_{1}\left(\operatorname{Tor}_{0}^{\mathcal{O}_{X}}\left(\mathcal{Q}, \mathcal{O}_{C}\right)\right)=c_{1}\left(\left.\mathcal{Q}\right|_{C}\right)
$$

So $\left.\mathcal{E}\right|_{C}$ is not slope-stable.

An immediate corollary is the following coarse criterion for checking slopestability with respect to $\gamma$.

Corollary 4.6. Let $\pi: C_{T} \rightarrow T$ be a family of smooth irreducible closed curves in $X$ with class $\gamma$. For $t \in T$ we write $C_{t}$ to denote $\pi^{-1}(t)$. Suppose $\mathcal{E}$ is a vector bundle on $X$ such that $\left.\mathcal{E}\right|_{C_{t}}$ is stable for all $t \in T$. If the curves in $C_{T}$ are dense in $X$ then $\mathcal{E}$ is stable with respect to the curve class $\gamma$.

Proof. Suppose for contradiction that $\mathcal{E}$ is unstable with respect to $\gamma$. Then there exists a torsion-free quotient $\mathcal{E} \rightarrow \mathcal{Q} \rightarrow 0$ with $\mu^{\gamma}(\mathcal{Q}) \leq \mu^{\gamma}(\mathcal{E})$. As $\mathcal{Q}$ is torsion-free, $\operatorname{Sing}(\mathcal{Q})$ has codimension $\geq 2$. The curves in $C_{T}$ are dense in $X$ so there is a $t \in T$ such that $C_{t}$ is not contained in $\operatorname{Sing}(\mathcal{Q})$. Then Proposition 4.5 guarantees that $\left.\mathcal{E}\right|_{C_{t}}$ is not stable, which contradicts our hypothesis.

Proposition 4.5 can be adjusted so that Corollary 4.6 also holds if stability is replaced by semistability. As a consequence we prove the following basic result 
about slope-stable vector bundles, which we have already used in the proof of Theorem A.

Proposition 4.7. Let $X$ and $Y$ be smooth projective varieties of dimension $d$ and $e$, respectively. Let $H_{X}$ be an ample divisor on $X$ and let $H_{Y}$ be an ample divisor on $Y$. Let $p_{1}$ denote the projection from $X \times Y$ to $X$ and $p_{2}$ the projection from $X \times Y$ to $Y$. If $\mathcal{E}$ is a vector bundle on $X$ which is slope-stable with respect to $H_{X}$, then $p_{1}^{*}(\mathcal{E})$ is slope-stable on $X \times Y$ with respect to the ample divisor $p_{1}^{*}\left(H_{X}\right)+p_{2}^{*}\left(H_{Y}\right)$.

Proof. By [Mehta and Ramanathan 1984, Theorem 4.3] if $k \gg 0$ and $C$ is a general curve which is a complete intersection of divisors linearly equivalent to $k H_{X}$ then $\left.\mathcal{E}\right|_{C}$ is stable. Let $F \subset\left|k H_{X}\right|^{d-1}$ be the open subset of the cartesian power of the complete linear series of $k H_{X}$ defined as

$$
F:=\left\{\left(H_{1}, \ldots, H_{d-1}\right) \in\left|k H_{X}\right|^{d-1} \mid C=H_{1} \cap \cdots \cap H_{d-1}\right.
$$

is a smooth complete intersection curve and $\left.\mathcal{E}\right|_{C}$ is stable\}.

We write $C_{F}$ for the natural family of smooth curves in $X$ parametrized by $F$. Likewise, the fiber product $C_{F} \times{ }_{F}(F \times Y)$ is naturally a family of smooth curves in $X \times Y$ parametrized by $F \times Y$. The image of $C_{F} \times{ }_{F}(F \times Y)$ in $X \times Y$ is dense, and for any $(f, y) \in F \times Y$ the restriction of $p_{1}^{*}(\mathcal{E})$ to $C_{(f, y)}$ is stable. Therefore, by Corollary $4.6, p_{1}^{*}(\mathcal{E})$ is stable with respect to the numerical class of $C_{(f, y)}$, which we denote by $\gamma$.

For $l \gg 0$ the divisor $l H_{Y}$ is very ample on $Y$ and a general complete intersection of divisors linearly equivalent to $l H_{Y}$ is smooth. Let $G \subset\left|l H_{Y}\right|^{e-1}$ be the open subset of the cartesian power of the complete linear series of $l H_{Y}$ defined as

$$
G:=\left\{\left(H_{1}, \ldots, H_{e-1}\right) \in\left|l H_{Y}\right|^{e-1} \mid H_{1} \cap \cdots \cap H_{e-1}\right.
$$

is a smooth complete intersection curve\}.

As before, there is a natural family $D_{G}$ of smooth curves in $Y$ parametrized by $G$. The fiber product $D_{G} \times{ }_{G}(X \times G)$ is a family of smooth curves in $X \times Y$ parametrized by $X \times G$. For $(x, g) \in X \times G$ the restriction of $p_{1}^{*}(\mathcal{E})$ to $D_{(x, g)}$ is a direct sum of trivial bundles, thus the restriction is semistable. Therefore, by applying Corollary 4.6 in the semistable case, $p_{1}^{*}(\mathcal{E})$ is semistable with respect to the curve class of $D_{(x, g)}$, which we denote by $\delta$.

Finally,

$$
\left(p_{1}^{*} H_{X}+p_{2}^{*} H_{Y}\right)^{d+e-1}=\left(\begin{array}{c}
d+e-1 \\
e
\end{array}\right) \frac{\left(H_{Y}\right)^{e}}{k^{d-1}} \cdot \gamma+\left(\begin{array}{c}
d+e-1 \\
d
\end{array}\right) \frac{\left(H_{X}\right)^{d}}{l^{e-1}} \cdot \delta .
$$

Thus, by Lemma $4.4, p_{1}^{*}(\mathcal{E})$ is slope-stable with respect to $p_{1}^{*}\left(H_{X}\right)+p_{2}^{*}\left(H_{Y}\right)$.

This completes the proof of Theorem A. We now give a proof of the perturbation argument. The idea is to use [Greb et al. 2016, Theorem 3.4] on openness of 
stability along with the fact that the natural Chow divisors are lef in the sense of [de Cataldo and Migliorini 2002, Definition 2.1.3].

Proposition 4.8. Let $H$ be a nef divisor and $A$ an ample $\mathbb{Q}$-divisor on a normal complex projective variety $X$. Suppose $\mathcal{E}$ is a rank-r torsion-free sheaf on $X$ which is slope-stable with respect to the class of $H^{d-1}$. Assume

$$
-\cap H^{d-2}: N^{1}(X)_{\mathbb{R}} \rightarrow N_{1}(X)_{\mathbb{R}}, \quad \xi \mapsto \xi \cdot H^{d-2}
$$

is an isomorphism. Then $\mathcal{E}$ is stable with respect to $H+\epsilon A$ for $\epsilon$ sufficiently small.

This implies that we can perturb our Chow polarization to obtain stability of tautological bundles with respect to nearby ample divisors.

Corollary 4.9. If $\mathcal{E}$ is a vector bundle on a smooth projective surface $S$ which is stable with respect to an ample divisor $H$, then $\mathcal{E}^{[n]}$ is stable with respect to an ample divisor near the Chow divisor $H_{n}$.

Proof of Corollary 4.9. By [de Cataldo and Migliorini 2002, Theorem 2.3.1] we know $H_{n}$ is lef, so $\mathcal{E}^{[n]}$ and $H_{n}$ satisfy the conditions of Proposition 4.8. Therefore, $\mathcal{E}^{[n]}$ is stable with respect to ample divisors close to $H_{n}$.

Proof of Proposition 4.8. Identifying the tangent space of a vector space with the vector space, the derivative of the $(d-1)$-st power map $N^{1}(X)_{\mathbb{R}} \rightarrow N_{1}(X)_{\mathbb{R}}$ at $H$ is given by

$$
-\cap(d-1) H^{d-2}: N^{1}(X)_{\mathbb{R}} \rightarrow N_{1}(X)_{\mathbb{R}} .
$$

The assumption that the intersection with the $H^{d-2}$ map is an isomorphism implies that the $(d-1)$-st power map is locally an isomorphism.

It follows from [Greb et al. 2016, Theorem 3.4] that there is a nonempty convex open set $U \subset N_{1}(X)_{\mathbb{R}}$ whose closure contains $\left[H^{d-1}\right]$ such that, for all $\gamma \in U, \mathcal{E}$ is stable with respect to $\gamma$. More precisely, if $\delta \in N_{1}(X)_{\mathbb{R}}$ represents the $(d-1)$-st power of an ample divisor then $\mathcal{E}$ is stable with respect to the perturbed curve class $\left[H^{d-1}\right]+\epsilon \cdot \delta$ for $\epsilon$ sufficiently small. By estimating the $(d-1)$-st power map by its derivative (which is an isomorphism at $H$ ) and by our ability to perturb linearly towards ample curve classes, we see that, for small enough $\epsilon,(H+\epsilon A)^{d-1}$ maps into U. Therefore, for $\epsilon$ sufficiently small, $\mathcal{E}$ is stable with respect to $H+\epsilon A$.

\section{Acknowledgements}

I am grateful to my advisor, Robert Lazarsfeld, who suggested the project and directed me in productive lines of thought. I am also thankful for conversations and correspondence with Lawrence Ein, Roman Gayduk, Daniel Greb, Julius Ross, Giulia Saccà, Ian Shipman, Brooke Ullery, Dingxin Zhang, and Xin Zhang. This paper is a substantial revision of a previous preprint. I would finally like to thank the referees for thoroughly reviewing the paper and offering helpful suggestions. 


\section{References}

[Arnold 1971] V. I. Arnold, "Matrices depending on parameters", Uspehi Mat. Nauk 26:2(158) (1971), 101-114. In Russian; translated in Russ. Math. Surv. 26:2 (1971), 29-43. MR Zbl

[Aubin 1976] T. Aubin, "Équations du type Monge-Ampère sur les variétés kähleriennes compactes", C. R. Acad. Sci. Paris Sér. A-B 283:3 (1976), Aiii, A119-A121. MR Zbl

[Beauville et al. 1989] A. Beauville, M. S. Narasimhan, and S. Ramanan, "Spectral curves and the generalised theta divisor", J. Reine Angew. Math. 398 (1989), 169-179. MR Zbl

[Bejleri and Stapleton 2016] D. Bejleri and D. Stapleton, "The tangent space of the punctual Hilbert scheme", preprint, 2016. arXiv

[Buchweitz et al. 2009] R.-O. Buchweitz, W. Ebeling, and H.-C. Graf von Bothmer, "Low-dimensional singularities with free divisors as discriminants", J. Algebraic Geom. 18:2 (2009), 371-406. MR $\mathrm{Zbl}$

[de Cataldo and Migliorini 2002] M. A. A. de Cataldo and L. Migliorini, "The hard Lefschetz theorem and the topology of semismall maps", Ann. Sci. École Norm. Sup. (4) 35:5 (2002), 759-772. MR $\mathrm{Zbl}$

[Danila 2001] G. Danila, "Sur la cohomologie d'un fibré tautologique sur le schéma de Hilbert d'une surface", J. Algebraic Geom. 10:2 (2001), 247-280. MR Zbl

[Donaldson 1985] S. K. Donaldson, "Anti self-dual Yang-Mills connections over complex algebraic surfaces and stable vector bundles", Proc. London Math. Soc. (3) 50:1 (1985), 1-26. MR Zbl

[Ellingsrud and Strømme 1993] G. Ellingsrud and S. A. Strømme, "Towards the Chow ring of the Hilbert scheme of $\mathbb{P}^{2}$ ", J. Reine Angew. Math. 441 (1993), 33-44. MR Zbl

[Fogarty 1973] J. Fogarty, "Algebraic families on an algebraic surface, II: The Picard scheme of the punctual Hilbert scheme”, Amer. J. Math. 95 (1973), 660-687. MR Zbl

[Fulton 1998] W. Fulton, Intersection theory, 2nd ed., Ergebnisse der Mathematik und ihrer Grenzgebiete. 3. Folge. A Series of Modern Surveys in Mathematics 2, Springer, Berlin, 1998. MR $\mathrm{Zbl}$

[Greb and Toma 2013] D. Greb and M. Toma, "Compact moduli spaces for slope-semistable sheaves", preprint, 2013. arXiv

[Greb et al. 2016] D. Greb, S. Kebekus, and T. Peternell, "Movable curves and semistable sheaves", Int. Math. Res. Not. 2016:2 (2016), 536-570. MR Zbl

[Kobayashi 1987] S. Kobayashi, Differential geometry of complex vector bundles, Publications of the Mathematical Society of Japan 15, Princeton University Press, Iwanami Shoten, Tokyo, 1987. MR $\mathrm{Zbl}$

[Lehn 1998] M. Lehn, “On the cotangent sheaf of Quot-schemes”, Internat. J. Math. 9:4 (1998), 513-522. MR Zbl

[Lehn 1999] M. Lehn, "Chern classes of tautological sheaves on Hilbert schemes of points on surfaces”, Invent. Math. 136:1 (1999), 157-207. MR Zbl

[Mehta and Ramanathan 1984] V. B. Mehta and A. Ramanathan, "Restriction of stable sheaves and representations of the fundamental group", Invent. Math. 77:1 (1984), 163-172. MR Zbl

[Mistretta 2006] E. C. Mistretta, Some constructions around stability of vector bundles on projective varieties, Ph.D. thesis, Université Paris Diderot, Paris 7, 2006, available at http://www.math.unipd.it/ ernesto/pub/tesi.pdf.

[Nakajima 1999] H. Nakajima, Lectures on Hilbert schemes of points on surfaces, University Lecture Series 18, American Mathematical Society, Providence, RI, 1999. MR Zbl 
[Okounkov 2014] A. Y. Okounkov, "Hilbert schemes and multiple $q$-zeta values", Funktsional. Anal. i Prilozhen. 48:2 (2014), 79-87. MR Zbl

[Scala 2009] L. Scala, "Cohomology of the Hilbert scheme of points on a surface with values in representations of tautological bundles", Duke Math. J. 150:2 (2009), 211-267. MR Zbl

[Schlickewei 2010] U. Schlickewei, "Stability of tautological vector bundles on Hilbert squares of surfaces", Rend. Semin. Mat. Univ. Padova 124 (2010), 127-138. MR Zbl

[Wandel 2013] M. Wandel, "Tautological sheaves: Stability, moduli spaces and restrictions to generalised Kummer varieties”, preprint, 2013. arXiv

[Wandel 2014] M. Wandel, "Stability of tautological bundles on the Hilbert scheme of two points on a surface”, Nagoya Math. J. 214 (2014), 79-94. MR Zbl

Communicated by David Eisenbud

Received 2015-06-28 Revised 2016-04-28 Accepted 2016-05-28

david.stapleton@stonybrook.edu Department of Mathematics, Stony Brook University,

Math Tower 2118, Stony Brook, NY 11794, United States 


\section{Algebra \& Number Theory}

msp.org/ant

\section{EDITORS}

MANAGING EDITOR

Bjorn Poonen

Massachusetts Institute of Technology

Cambridge, USA

\author{
EDITORIAL BOARD CHAIR \\ David Eisenbud \\ University of California \\ Berkeley, USA
}

BOARD OF EDITORS

$\begin{aligned} \text { Dave Benson } & \text { University of Aberdeen, Scotland } & \text { Susan Montgomery } & \text { University of Southern California, USA } \\ \text { Richard E. Borcherds } & \text { University of California, Berkeley, USA } & \text { Shigefumi Mori } & \text { RIMS, Kyoto University, Japan } \\ \text { John H. Coates } & \text { University of Cambridge, UK } & \text { Raman Parimala } & \text { Emory University, USA } \\ \text { J-L. Colliot-Thélène } & \text { CNRS, Université Paris-Sud, France } & \text { Jonathan Pila } & \text { University of Oxford, UK } \\ \text { Brian D. Conrad } & \text { Stanford University, USA } & \text { Anand Pillay } & \text { University of Notre Dame, USA } \\ \text { Hélène Esnault } & \text { Freie Universität Berlin, Germany } & \text { Victor Reiner } & \text { University of Minnesota, USA } \\ \text { Hubert Flenner } & \text { Ruhr-Universität, Germany } & \text { Peter Sarnak } & \text { Princeton University, USA } \\ \text { Sergey Fomin } & \text { University of Michigan, USA } & \text { Joseph H. Silverman } & \text { Brown University, USA } \\ \text { Edward Frenkel } & \text { University of California, Berkeley, USA } & \text { Michael Singer } & \text { North Carolina State University, USA } \\ \text { Andrew Granville } & \text { Université de Montréal, Canada } & \text { Vasudevan Srinivas } & \text { Tata Inst. of Fund. Research, India } \\ \text { Joseph Gubeladze } & \text { San Francisco State University, USA } & \text { J. Toby Stafford } & \text { University of Michigan, USA } \\ \text { Roger Heath-Brown } & \text { Oxford University, UK } & \text { Ravi Vakil } & \text { Stanford University, USA } \\ \text { Craig Huneke } & \text { University of Virginia, USA } & \text { Michel van den Bergh } & \text { Hasselt University, Belgium } \\ \text { Kiran S. Kedlaya } & \text { Univ. of California, San Diego, USA } & \text { Marie-France Vignéras } & \text { Université Paris VII, France } \\ \text { János Kollár } & \text { Princeton University, USA } & \text { Kei-Ichi Watanabe } & \text { Nihon University, Japan } \\ \text { Yuri Manin } & \text { Northwestern University, USA } & \text { Efim Zelmanov } & \text { University of California, San Diego, USA } \\ \text { Philippe Michel } & \text { École Polytechnique Fédérale de Lausanne } & \text { Shou-Wu Zhang } & \text { Princeton University, USA }\end{aligned}$

PRODUCTION

production@msp.org

Silvio Levy, Scientific Editor

See inside back cover or msp.org/ant for submission instructions.

The subscription price for 2016 is US $\$ 290$ /year for the electronic version, and $\$ 485 /$ year (+\$55, if shipping outside the US) for print and electronic. Subscriptions, requests for back issues and changes of subscribers address should be sent to MSP.

Algebra \& Number Theory (ISSN 1944-7833 electronic, 1937-0652 printed) at Mathematical Sciences Publishers, 798 Evans Hall \#3840, c/o University of California, Berkeley, CA 94720-3840 is published continuously online. Periodical rate postage paid at Berkeley, CA 94704, and additional mailing offices.

ANT peer review and production are managed by EditFLow ${ }^{\circledR}$ from MSP.

\section{PUBLISHED BY}

- mathematical sciences publishers

nonprofit scientific publishing

http://msp.org/

() 2016 Mathematical Sciences Publishers 


\section{Algebra \& Number Theory}

Volume $10 \quad$ No. $6 \quad 2016$

Modular elliptic curves over real abelian fields and the generalized Fermat equation $x^{2 \ell}+y^{2 m}=z^{p}$

SAMUELE ANNI and SAMIR SIKSEK

Geometry and stability of tautological bundles on Hilbert schemes of points

DAVID STAPLETON

Anabelian geometry and descent obstructions on moduli spaces

Stefan PATRIKIS, José Felipe Voloch and Yuri G. Zarhin

On the local Tamagawa number conjecture for Tate motives over tamely ramified fields JAY DAIGLE and MATTHIAS FLACH

Heegner divisors in generalized Jacobians and traces of singular moduli

JAN HENDRIK BRUINIER and YINGKUN LI

On 2-dimensional 2-adic Galois representations of local and global fields VYTAUTAS PAŠKŪNAS

A probabilistic Tits alternative and probabilistic identities

Michael LARSEN and ANER SHALEV 